\title{
Adaptation of Hoarding Rating Scale Interview in Urdu for Use in Pakistan
}

FAUZIA MALIK, ANILA KAMAL

National Institute of Psychology, Quaid i Azam University, Islamabad, Pakistan

Correspondence to Fauzia Malik, Email: fauziamalick@gmail.com, Contact: 03015782645. H No. 163, Block A, Guldasht Town, Lahore.

\begin{abstract}
Background: Hoarding Rating Scale Interview (HRS-I) is a brief five item instrument to assess symptoms of hoarding in both clinical and non-clinical population.

Aim: To adapt and validate HRS-I in Urdu language for its convenient use in Pakistan.

Methods: Sample consisted of 112 adults from both clinical and a control group. The scale was translated in Urdu following standard translation procedure and data was collected to determine the psychometric properties of translated version. Reliability and validity estimates were established using different statistical analysis.

Results: Cronbach alpha value (.82) suggested an acceptable level of internal consistency. Factor structure was found to be consistent with original English version of HRS-I and correlations were found to be high for like subscales than other subscales except for a positive but non-significant correlation between acquisition item of HRS-I and acquisition subscale of SI-R. Though it significantly correlated with total scale score on HRS-I.

Conclusion: The results of the study provide evidence for preliminary acceptable psychometric properties of Urdu version of Hoarding Rating Scale-Interview and proves it to be a time efficient and promising tool to assess hoarding in cultural context of Pakistan.

MeSH words: HRS-I, Validation study, Assessment of Hoarding, Cultural adaptation.
\end{abstract}

\section{INTRODUCTION}

Hoarding is categorized as accumulation of large number of possessions and difficulty discarding most of the items irrespective of their real worth. This results in severe disorganization and cluttering of living spaces making it difficult for their intended use. Consequently it brings in distress and impairment in daily life activities. It has now been classified as a standalone disorder in recent edition of Diagnostic and Statistical Manual of Mental Disorders ${ }^{1}$. Its onset is thought to be in early childhood and has a progressive course $^{2}$ with a lifetime prevalence now considered to be 2 and $5 \%{ }^{3}$.

There are a number of instruments available to assess different aspects of hoarding such as acquiring, difficulty discarding, level of clutter, and associated distress with these features. For instance, Saving Inventory Revised ${ }^{4}$ which is a 23 item self-report measure to assess different dimensions of hoarding. Clutter Image Rating ${ }^{5}$ that provides a pictorial illustration of different levels of clutter to assess hoarding severity and monitor treatment progress over time. Also Activities of Daily Living ${ }^{2}$ for Hoarding to measure interference caused by clutter and Saving Cognitions Inventory 6 to assess beliefs and attitudes associated with discarding task.

In the same way Hoarding Rating Scale Interview ${ }^{7}$ is another widely used tool that is used to assess hoarding. It is a brief instrument consisting of five items based on criteria of hoarding disorder given by DSM-V. It asks about level of clutter, difficulty in discarding possessions, acquiring problem, and associated distress and impairment. The five symptoms of hoarding are systematically measured by HRS-I on a 9-point Likert type scale. It has established psychometric properties with an

Received on 24-11-2020

Accepted on 14-04-2021 alpha value of .97 as a measure of internal consistency and an excellent test-retest reliability. It has also shown high correlation values when administered with other instruments providing an evidence for convergent and discriminant validity.

Given the high prevalence of hoarding across different regions of the world and understanding the importance of test adaptations ${ }^{8}$ while using them in different cultures we find it valuable to translate and adapt HRS-I in Urdu for its use in Pakistan. As it can be used both as an interview and as a self-report measure and being a time efficient tool it would be of great benefit for clinicians and researchers alike to have HRS-I in Urdu for furthering the research on hoarding in Pakistan. The present study therefore aims to translate and adapt HRS-I into Urdu using standard procedure ${ }^{9}$ for test adaptation. Purpose of the study is to examine the reliability and validity of the adapted version by observing factor structure, internal consistency, and correlation with other measures.

\section{METHODS}

Participants: Participants consisted of 112 adults ( $\geq 18 y r s$ ), 55 of whom were clinical outpatients from different hospitals of Rawalpindi and Islamabad. Patients suffering affective disorders of depression, anxiety, and ocd only were considered for the present study. 57 participants as non-clinical controls without history of any psychopathology were also part of the study. All the participants were able to read and understand the study questionnaires. Informed consent was taken from all the study participants and right to withdraw at any time was given.

Measures

Hoarding Rating Scale Interview (HRS-I): It is a five item instrument that measures the different dimensions of hoarding including clutter, difficulty discarding, excessive 
acquisition, distress and impairment caused by the behavior. It is a nine point $(0=$ none to $8=$ extreme) Likert type scale and a total score can be calculated by adding score on all the five items.

Saving Inventory Revised Urdu Version (SI-R-U): SI-R$\mathrm{U}^{10}$ is a 20 item modified version of original SI-R (Frost et al, 2004) which is a self-report measure consisting of 23 items used to assess hoarding among both clinical and non-clinical groups. It has a five point Likert scale format ranging from zero (none) to four (almost all/extreme). It has three subscales measuring excessive acquisition, clutter and difficulty discarding. It has shown good internal consistency ( $\alpha=.82$ to .63) and convergent and discriminant validity.

Obsessive Compulsive Inventory Revised (OCI-R): It is an 18 item self-report instrument ${ }^{11}$ that measures OCD symptoms including Obsessing, Neutralizing, Ordering, Checking, Washing, and Hoarding dimensions. It keeps strong internal consistency $(\alpha=.84)$ and has shown good test-retest reliability and validity.

Depression Anxiety Stress Scale (DASS): DASS ${ }^{12}$ comprises of three subscales that measure the negative emotional states of depression, anxiety, and stress. It is a four point Likert scale that assesses each state over the past week. Score is expressed as mild, moderate, severe, and extremely severe. It has shown to be a reliable and valid measure over time. A shorter version consisting of 21 items is used for this study.

Procedure: HRS-I was first translated into Urdu using translation/back translation approach. Data was then collected on all study measures from both clinical and nonclinical groups after briefly explaining the purpose of study and taking written informed consent from the participants. Trained interviewer's administered the protocols and it took approximately 20 to 30 minutes to complete the study measures including demographic sheet asking about age, gender, education, family system, etc. However most of the participants from non-clinical group were provided with the questionnaire packet and they completed it themselves. From student sample data was collected in groups.

Translation of Hoarding Rating Scale Interview: Using standard procedure for cross cultural adaptation given by Brislin ${ }^{9}$ HRS-I was translated into Urdu language. For the purpose it was translated from English (source language) into Urdu (target language) by five bilingual experts focusing on maximization of content similarity and conceptual equivalence. Committee approach was then sought and most consistent translations were carefully chosen. The finalized translation was again given to five other experts keeping the understanding of both the languages for translating it back to source language that is English. Next another group of experts examined the translations to finalize the back translated version by looking at translation inaccuracies and cross-checking the items with original English version of HRS-I. The finalized Urdu translated version was then field tested to examine its psychometric properties.
Statistical analysis: Data was analyzed using SPSS 20 and AMOS 22 statistical package. Confirmatory factor analysis was used to verify the factor model as derived in prior studies on US/European samples. Using Maximum likelihood different indices $(\mathrm{X} 2>.05, \mathrm{CFI}>.9$, RMSEA $\leq$ .05 , SRMR <.1, minimum factor loading > .3) were evaluated for ascertaining a good fit. Reliability and validity estimates were examined with calculating Cronbach's alpha and Pearson correlation coefficients respectively.

\section{RESULTS}

Sample Characteristics: The age ranged between 18 to 50 years $(M=32.9)$ with $61 \%$ of sample being Males. $60 \%$ of the participants were married and most of them (67\%) belonged to nuclear family system. From among clinical patients $60 \%$ were suffering depression, $21.6 \%$ have anxiety disorder diagnosis while $10 \%$ were OCD sufferers. Non- clinical group appears to be more involved in hoarding (12.3\%) as compare to clinical group in which $7.3 \%$ seems to be hoarders. Females (13.5\%) show more of hoarding characteristics as compare to male (6.7\%) participants.

Factor Structure: Confirmatory factor analysis (Fig. 1) revealed a uni-dimension structure. Different indices were all indicative of a good model fit with $X 2=6.48, \mathrm{CFI}=.99$, $\mathrm{TLI}=.98$ and $\mathrm{RMSEA}=.05$. Factor loadings were also high ranging from .58 to .81 .

Reliability and Validity Estimates: Cronbach's a value for the scale was found to be .82 . Inter-item correlations were calculated and found between .36 and .60 while correlation between each item and total score ranged from .68 and .62. Moderate correlations between items and strong correlations of each item with total score specify that indicators though distinct from each other belong to same latent variable and are suggestive of internal consistency of the scale.

Receiver operating characteristic analysis (Fig. 2) was done to measure HRS's discriminative power to correctly identify hoarders and non-hoarders. The area under the curve for scale items and total score was item 1 (Clutter) $=.95$, item 2 (Difficulty discarding) $=.91$, item 3 (Acquisition) $=.76$, item 4 (Distress) $=.84$, item 5 (Impairment $)=.93$ and .97 for total score suggesting an overall good discrimination.

Pearson correlations were calculated between HRS-I scores and measures of interest as shown in Table 1. Total score as well as score on each item of HRS-I correlated significantly with corresponding subscale of SI-R-U except for Acquisition item that has shown a non-significant correlation with acquisition subscale of SI-R-U. However the strength of correlations is found to be modest. Similarly HRS-I total and each item has shown significant positive correlation with measures of hoarding, depression, and anxiety. Only acquiring item has shown a non-significant correlation with measure of depression. However the strongest correlation is found with hoarding subscale of OCI-R. 
Table 1: Pearson correlation of HRS-I with measures of interest $(\mathrm{N}=112)$

\begin{tabular}{|c|c|c|c|c|c|c|}
\hline 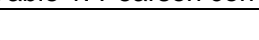 & Clutter & Difficulty Discarding & Acquisition & Distress & Impairment & Total \\
\hline \multicolumn{7}{|l|}{ SI-R-U } \\
\hline Total & $.38^{* *}$ & $.35^{\star \star}$ & $23^{*}$ & $.39^{\star \star}$ & $.32^{* *}$ & $.43^{* *}$ \\
\hline Clutter & $.34^{\star *}$ & $.28^{* *}$ & $.21^{*}$ & $.35^{\star *}$ & $.26^{\star *}$ & $.38^{* *}$ \\
\hline Difficulty Discarding & $.32^{* \star}$ & $.29^{\star \star}$ & $.18^{*}$ & $.27^{\star \star}$ & $.21^{*}$ & $.33^{* *}$ \\
\hline Acquisition & $.30^{\star *}$ & $.32^{\star \star}$ & .17 & $.33^{\star *}$ & $.32^{\star *}$ & $.37^{* *}$ \\
\hline OCl-R-H & $.23^{*}$ & $.43^{\star \star}$ & $.38^{\star \star}$ & $.45^{\star \star}$ & $.31^{\star \star}$ & $.47^{\star \star}$ \\
\hline DASS- D & $.31^{\star *}$ & $21^{*}$ & .14 & $.36^{\star \star}$ & $.32^{\star \star}$ & $.35^{\star \star}$ \\
\hline DASS-A & $.27^{* \star}$ & $23^{*}$ & $.26^{* *}$ & $.42^{* *}$ & $.43^{\star \star}$ & $.42^{* *}$ \\
\hline
\end{tabular}

${ }^{* *} p<.05$

Note: SI-R-U= Saving Inventory Revised Urdu version, OCI-R-H =Hoarding subscale of Obsessive Compulsive Inventory Revised, DASS-D =Depression subscale of Depression Anxiety Stress scale, DASS-A = Anxiety subscale of Depression Anxiety Stress scale.

Figure 1: Confirmatory factor Analysis of HRS-I

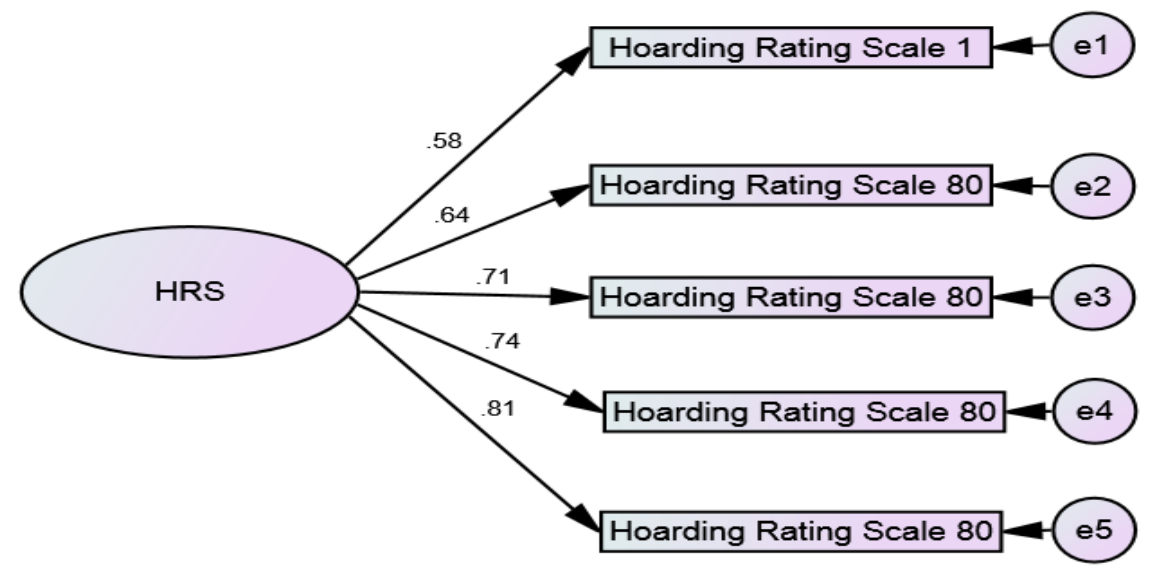

Figure 2: Receiver Operating Characteristic Curve of HRS-I

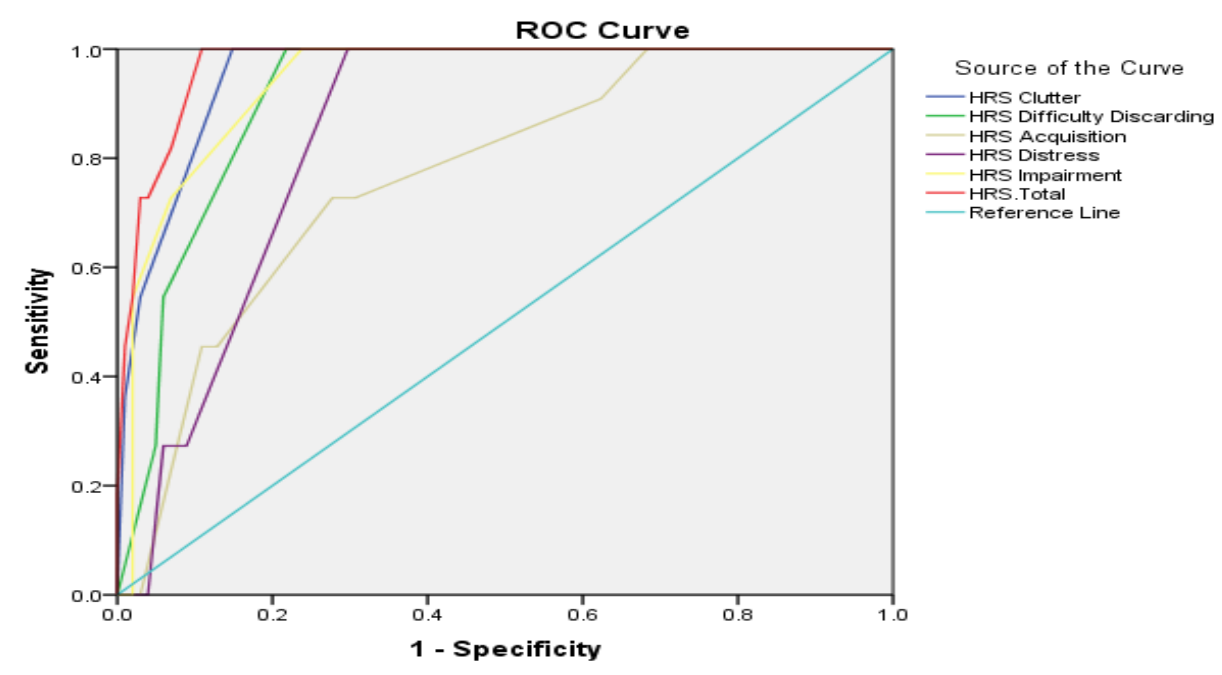

\section{DISCUSSION}

The present study is the first one to adapt and validate Hoarding Rating Scale-Interview (HRS-I) into Urdu that is National language of Pakistan. HRS-I is a useful instrument to assess hoarding and can be used both as a self-report measure and in interview format. Its availability in Urdu is therefore considered beneficial for evaluating hoarding behavior in both clinical and non-clinical patients. Our study demonstrates the preliminary evidence of reliability and validity of Urdu version of HRS-I as indicated by acceptable values of alpha coefficient and correlation coefficients between HRS-I items and other measures of interest. 
A consideration of factor structure maintained the unidimensional nature of the instrument as suggested by the original study done by Tolin, Frost, and Steketee ${ }^{7}$. All the items have shown acceptable factor loading of above .3. Results of the present study demonstrate good internal consistency of the scale as shown by an alpha value of .82 and satisfactory inter item correlations. It also exhibited good discrimination power as displayed by Receivers Operating Characteristic analysis. Validity estimates of the scale as measured by the correlation between HRS-I and other measures were also found to be in acceptable range. These results are consistent with previous studies ${ }^{13,14,15}$ on adaptation of hoarding rating scale interview.

Besides notable is the non-significant correlation between acquisition subscale of SI-R-U and item measuring acquisition on HRS-I. Though there is a significant correlation between acquiring subscale of SI-R$U$ and total score on HRS-I. We find no apparent reason for this unexpected outcome however a possible explanation could be that SI-R-U has more diverse items concerning acquiring that are associated with distress and urge related content while HRS-I acquiring item measures level of acquiring behavior. Yet it needs further evaluation. Likewise a non-significant correlation between acquiring and measure of depression is contrary to findings of previous research ${ }^{13}$.

\section{CONCLUSION}

To conclude the current study provides evidence for preliminary acceptable psychometric properties of Urdu version of Hoarding Rating Scale-Interview. It appears to be a time efficient and promising tool to assess hoarding in cultural context of Pakistan. However the study is limited in its generalizability because of small sample size and absence of participants with known hoarding disorder. Also retest reliability was not assessed in this study. Further research is therefore needed to address these issues and to examine the findings that are inconsistent with the previous research.

Acknowledgements: Foremost I would extend my gratitude to Higher Education Commission (HEC) of Pakistan who gave me scholarship to pursue my Ph.D. degree. Second I would acknowledge role of my supervisor Dr. Anila Kamal for her continued guidance. To all the bilingual experts for their valuable time and help in Translation process. To committee members for evaluation of translations and their expert opinion and insight. And definitely to all the participants of my study who invested time and energy to complete the study measures.

Source of Support: This research article is from my Ph.D work and Higher Education Commission of Pakistan gave me scholarship to pursue my degree. Except this no other funding is involved in this research.

\section{REFERENCES}

1. American Psychiatric Association. Diagnostic and statistical manual of mental disorders (DSM-5®). American Psychiatric Pub; 2013 May 22.

2. Grisham JR, Frost RO, Steketee G, Kim HJ, Hood S. Age of onset of compulsive hoarding. Journal of anxiety disorders. 2006 Jan $1 ; 20(5): 675-86$.

3. Iervolino AC, Perroud N, Fullana MA, Guipponi M, Cherkas L, Collier $\mathrm{DA}$, Mataix-Cols D. Prevalence and heritability of compulsive hoarding: a twin study. American Journal of Psychiatry. 2009 Oct;166(10):115661.

4. Frost RO, Steketee G, Grisham J. Measurement of compulsive hoarding: saving inventory-revised. Behaviour research and therapy. 2004 Oct 1;42(10):1163-82.

5. Frost RO, Steketee G, Tolin DF, Renaud S. Development and validation of the clutter image rating. Journal of Psychopathology and Behavioral Assessment. 2008 Sep 1;30(3):193-203.

6. Steketee G, Frost RO, Kyrios M. Cognitive aspects of compulsive hoarding. Cognitive Therapy and Research. 2003 Aug 1;27(4):463-79.

7. Tolin DF, Frost RO, Steketee G. A brief interview for assessing compulsive hoarding: the Hoarding Rating Scale-Interview. Psychiatry research. 2010 Jun 30;178(1):147-52.

8. Hambleton RK, Patsula L. Adapting tests for use in multiple languages and cultures. Social indicators research. 1998 Nov 1;45(1-3):153-71.

9. Brislin RW. Comparative research methodology: Cross-cultural studies. International journal of psychology. 1976 Jan 1;11(3):215-29.

10. Malik F, Kamal A. (2020). Validation of Saving Inventory Revised and Consideration of Associated Factors in Cultural Context of Pakistan. Unpublished Manuscript. 2020

11. Foa EB, Huppert JD, Leiberg S, Langner R, Kichic R, Hajcak G, Salkovskis PM. The Obsessive-Compulsive Inventory: development and validation of a short version. Psychological assessment. 2002 Dec;14(4):485

12. Lovibond PF, Lovibond SH. The structure of negative emotional states: Comparison of the Depression Anxiety Stress Scales (DASS) with the Beck Depression and Anxiety Inventories. Behaviour research and therapy. 1995 Mar 1;33(3):335-43.

13. Faraci $\mathrm{P}$, Perdighe $\mathrm{C}$, Del Monte $\mathrm{C}$, Saliani AM. Hoarding rating scaleinterview: reliability and construct validity in a nonclinical sample. International journal of psychology and psychological therapy. 2019;19(3):345-52.

14. Liu TW, Lam SC, Chung $\mathrm{MH}, \mathrm{Ho} \mathrm{KH}$. Adaptation and psychometric testing of the hoarding rating scale (HRS): a self-administered screening scale for epidemiological study in Chinese population. BMC psychiatry. 2020 Dec;20:1-0.

15. Tsuchiyagaito A, Horiuchi S, Igarashi T, Kawanori Y, Hirano Y, Yabe $H$, Nakagawa A. Factor structure, reliability, and validity of the Japanese version of the hoarding rating scale-self-report (hrs-sr-J). Neuropsychiatric disease and treatment. 2017;13:1235. 